Aus der Königlichen Universitätsklinik für Kehlkopf- und Nasenkrankheiten zu Budapest.

(Direktor Prof. Onodi.)

\title{
Eine indirekte Untersuchungsmethode des Kehlkopfes (Distractio Laryngis*)).
}

\author{
Von Dr. Aurelius Réthi.
}

Mit 6 Abbildungen.

Drei Punkte sind als Vorbedingungen des guten Gelingens des Operation aufzufassen: die entsprechende Güte der Instrumente, die Gewandtheit des Operateurs, und endlich die gute Zugänglichkeit des Operationsfeldes. Wenn nur eine dieser Bedingungen fehlt, ist die gelungene Lösung sehr in Frage gestellt. Je schärfer diese Bedingungen ausgeprägt sind, desto leichter hat es der Operateur.

Die zwei ersten Bedingungen will ich bei dieser Gelegenheit aus meiner Besprechung ausschalten, da erste eine individuelle Eigenschaft ist, und selbst die zweite auf hohem Niveau steht, die dritte Bedingung, d. h. die gute Zugänglichkeit, bedarf noch immer einer Verbesserung.

Lange Zeit hindurch war der Kehlkopfspiegel das einzige Instrument, welches uns einen Einblick in das Kehlkopfinnere gewährte. Dieser ermöglichte hauptsächlich seit der Einführung des Kokains die indirekte Kehlkopfoperation. Die von Kirstein und Killian empfohlene direkte Laryngoskopie gibt den Weg zu den direkten Operationen. Diese Operationsmethode konnte aber nicht als eine ideale bezeichnet werden.

Als ein Meilenstein ist die Killiansche Schwebelaryngoskopie zu betrachten. Wie bekannt, wird das Verfahren so ausgeübt, daß ein entsprechend konstruierter, hakenartiger Spatel in den Mund des auf seinem Rücken liegenden Patienten eingeführt, und in den Kehlkopf eingehakt wird. Der Spatel ist über dem Kopfe des Patienten aufgehängt. Der Kopf des Patienten wird jetzt losgelassen, wodurch der Kopf bloß an dem Spatel hängt. Infolge des Selbstgewichtes des Kopfes wird ein mächtiger Druck auf die Kehlkopfregion ausgeübt,

*) Am Lebenden demonstriert an der Tagung des Vereins Deutscher Laryngologen zu Kiel, Igr4. 
dadurch wird der Kehlkopf und die oberhalb des Kehlkopfes sitzenden Weichteile nach vorne, d. h. an dem liegenden Patienten nach aufwärts verschoben, wodurch die Zahnlücke und das Kehlkopflumen in eine gerade Linie gerät. So ist der Kehlkopf für unsere Operationsinstrumente direkt zugänglich.

Dieses Verfahren ist bei einem stattlichen Teil der Fälle sehr gut verwendbar. Das Verfahren hat aber leider einen Nachteil, und zwar daß seine Verwendung dem Patienten nicht nur unangenehm, sondern auch oft sehr schmerzhaft ist, so daß selbst Killian bei dem Kinde die allgemeine Narkose, bei dem Erwachsenen die Skopolaminnarkose empfiehlt.

Es ist selbstverständlich, daß wir bei einem Polyp des Stimmbandes, dessen indirekte Entfernung ganz schmerzlos ist, nicht die mit einer Skopolaminnarkose verbundene, oft schmerzhafte Schwebelaryngoskopie benüizen, da wir unsere Aufgabe mit der indirekten Methode ebenso gut lösen können. Kleinere Tumoren, mindergradige tuberkulöse Infiltrationen der Stimmbänder können ebenfalls indirekt operiert werden. Das Killiansche Verfahren ist in den Fällen indiziert, welche infolge ihrer großen Ausdehnung auf dem indirekten Wege nicht genügend radikal operiert werden können. Das Verfahren ist unentbehrlich bei allen operativen laryngealen Eingriffen der kleineren Kinder.

Zweifellos, die meisten Fälle werden indirekt operiert, trotzdem daß diese Methode nicht ganz leicht ist.

Was ist die Ursache, daB die indirekten Kehlkopfoperationen schwierig sind?

Die natürlichste und nahestehendste Antwort wäre, daß die Operation dadurch erschwert wird, daß wir mit Hilfe des Spiegels operieren, wir betrachten also das Operationsterrain in einem verkehrten Bilde. Diese Antwort ist aber nicht acceptierbar. Wenn wir einen Anfänger vor das Kehlkopfphantom setzen, so wird er nach einer kurzen Übung sehr leicht lernen, im Lichte des Kehlkopfspiegels mit einer Kehlkopfsonde jeden beliebigen Punkt der Stimmbänder pünktlich zu berïhren, auf das Stimmband gestellte Fremdkörper prompt zu entfernen. Wenn wir jetzt jenen Anfänger vor den lebendigen Kehlkopf setzen, so wird ihn bald sein bei dem Phantom geschaffenes Wissen im Stiche lassen.

Das Arbeiten am Lebenden ist also bedeutend schwieriger wie an dem Kehlkopfphantom, weil:

I. Die Stimmbänder des lebendigen Kehlkopfes nicht solche fixe Linien wie die des Phantoms bilden, da sie sich während der Ex- und Inspiration hin und her bewegen.

2. Wenn auch die Anästhesierung des Kehlkopfes vollkommen ist, können wir trotzdem in den meisten Fällen beobachten, daß die 
Stimmbänder sich nach einigen Berührungen krampfartig aneinander pressen.

3. Die Epiglottis legt sich auf den Kehlkopfeingang und bedeckt den größten Teil der Stimmbänder.

4. Die Zunge wölbt sich oft krampfartig vor.

5. Da wir mit einer Hand den Kehlkopfspiegel, mit der anderen das Instrument halten, so müssen wir das Hervorziehen der Zunge dem Patienten überlassen. Wenn eine stark entwickelte Hand des Patienten sich mit der Ungeschicklichkeit desselben paart, werden die Schwierigkeiten noch mehr erhöht.

Diese einzelnen unbedeutenden Schwierigkeiten resultieren die Schwierigkeiten der indirekten Operationsmethode.

Wenn wir nun wollen, daß die indirekte Larynxoperation leicht ausführbar werde, müssen wir ähnliche Verhältnisse schaffen, wie wir sie im Phantom finden.

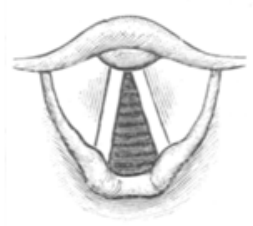

Abb. I.

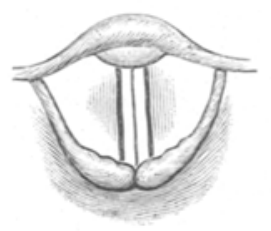

Abb. 2.

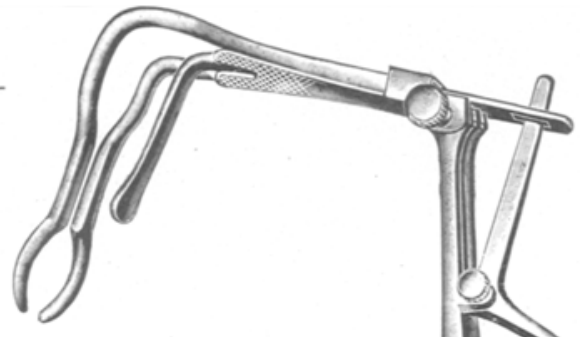

Abb. I zeigt die Glottis im offenen, Abb. 2 im geschlossenen Zustande. Diese Figuren demonstrieren uns, daß, wenn wir die geschlossene Glottis öffnen, und offen halten wollen, müssen wir auf drei Punkte einen Druck ausüben: auf die Wurzel der Epiglottis und auf die beiden Arygegenden.

Daraus folgt: wenn wir die Stimmbänder fixieren wollen, benötigen wir ein Instrument, welches diese drei Punkte voneinander fernhält, indem es auf die erwähnten drei Punkte einen entsprechenden Druck ausübt.

Nach vielen Versuchen ist es mir gelungen, ein entsprechendes Instrument zu konstruieren, welches die Schwierigkeiten der indirekten Larynxoperationen auf ein Minimum zu reduzieren imstande ist.

Das Instrument ist in der Abb.3zu sehen. Der dem Kopfe entsprechende Teil des Instrumentes, welches ich als Distraktor bezeichnen will, besteht aus drei Branchen, aus zwei hinteren und einer vorderen Die vordere ist breiter und kürzer, da sie bloß supraglottisch Platz nimmt 
und die Epiglottis nach vorn drückt, wodurch die ganze vordere Wand frei wird. Vor der Einführung des Instrumentes liegen die zwei hinteren Branchen dicht nebeneinander und neben der vorderen Branche, wenn aber das Instrument geschlossen wird, so entfernen sich die hinteren Branchen von der vorderen nach hinten zu, aber auch voneinander in der seitlichen Richtung. Die hinteren Branchen kommen subglottisch. Die Partie der hinteren Branchen, welche in dem Niveau der Stimmbänder Platz nimmt, verbreitet sich nach vorne zu einem Dreieck, wodurch die Glottis ad maximum geöffnet wird und die Stimmbänder fixiert werden. Das wird dadurch erreicht, daß die hinteren Branchen sich voneinander in der seitlichen Richtung entfernen, und einen mäßigen Druck auf die Muskelfortsätze der Gießknorpel ausüben. Da fernerhin die entsprechende Partie der hinteren Branchen nach vorne $\mathrm{zu}$ sich verbreitet, so rotiert sich der Aryknorpel nach der Seite zu, die Glottis ist dadurch ad maximum geöffnet, und die Stimmbänder sind fixiert. Da aber die hinteren Branchen sich auch von der vorderen Branche entfernen, wodurch die vordere Branche auf die Epilgottiswurzel und so indirekt auf den Schildknorpel, einen Druck ausübt, und so der vordere Anhaftspunkt der Stimmbänder nach vorne geschoben wird, während die hinteren Branchen den Ringknorpel und so den hinteren Anhaftspunkt der Stimmbänder nach hinten zu drücken, so werden die Stimmbänder angespannt.

Natürlich mußte dafür gesorgt werden, daß das Instrument nicht hinausfallen kann, daß es eine entsprechende Stütze bekomme. Die Fixation des Instrumentes an der hinteren Kehlkopfwand ist unmöglich, da diese Wand ganz glatt und steil ist, deshalb habe ich zu diesem Zwecke die seitlichen Wände ausgenützt.

Wie an dem nach meiner Methode gemachten sagittalen Röntgenbilde zu sehen ist (Abb. 4), bildet die seitliche Wand des Kehlkopfes unterhalb der Stimmbänder eine Vertiefung. Diese Vertiefung nützte ich aus, indem ich das untere Drittel der hinteren Branchen bogenartig konstruierte. Nach dem seitlichen Abgehen der hinteren Branchen legen sich die bogenartigen Krümmungen in die entsprechenden seitlichen Vertiefungen des Kehlkopfes, wodurch die Fixation des Instrumentes beinahe gesichert ist. Da aber die Branchen federn müssen, aus dem Grunde, damit durch den Druck eine Weichteilbeschädigung nicht entstehen könne, so könnte das Instrument bei eventuellem Würgen hinausfallen. Damit der Fall nicht vorkomme, wird eine entsprechende Mundsperre appliziert (Abb.5). Die Mundsperre hat noch den Vorteil, daß sie das horizontale Mundstück des Distraktors hinabdrückt, wodurch die Zunge ganz hinuntergedrückt und ein auffallend freier Einblick geschaffen wird.

Die oberhalb der Stimmbänder sitzende Partie der hinteren Bran- 
chen weichen voneinander $a b$, wodurch der freie Einblick gesichert wird.

Der Kopfteil des Distraktors geht in einem Winkel von Io7 Grad in den horizontalen Mundteil über, welcher einen breiten Zungenspatel besitzt, der Mundteil hat eine Fortsetzung in dem Griffe, an welchem ein Hebel angebracht ist. Wenn wir den Hebel hinunterdrücken, wird die vordere Branche, mit welcher der Hebel im Zusammenhang steht, nach vorne gezogen; dadurch keilt sich der an der unteren Fläche des horizontalen Teiles der hinteren Branche angebrachte längliche Keil zwischen die zweihinterenBranchen, die vorne aus einem Stücke ausgehen. Dadurch wird erreicht, daB die hinteren $B$ ranchen von der vorderen nach rückwärts und voneinander in der seitlichen Richtung entfernt werden. Wenn also der Hebel wieder aufgehoben wird, nähert sich die vordere Branche den hinteren, der Keil entfernt sich aus den hinteren Branchen, und da wie gesagt, die hinteren Branchen sich vorne vereinigen, legen sie sich infolge der Elastizität wieder zusammen.

Sehr wichtig ist es aber, daß wir vor der Applikation mit dem Schließen und Öffnen des Instrumentes ganz im Klaren sein sollen. Das Schließen, d. h. das Abdrücken des Hebels, ist ganz einfach. Den Griff halten wir in der rechten Hand, während der Daumen den Kopf des Hebels hinabdrückt. Da bei den verschiedenen Fällen die Schließung in verschiedenem Grade nötig ist, so wird das durch einen mit Zähnen versehenen Fixierhebel

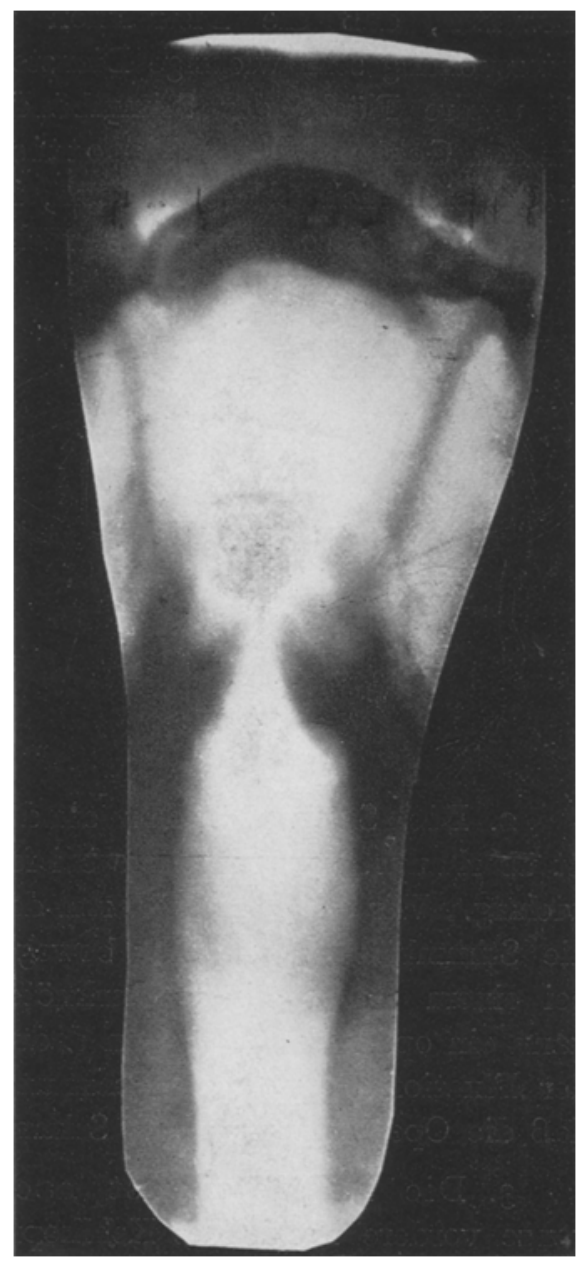

Abb. 4. erreicht. Die Zähne fixieren den eigentlichen Hebel in jedem beliebigen Grade. Das Öffnen des Instrumentes, d. h. das Heben des Hebels wird ganz einfach dadurch erreicht, da $\beta$ wir mit dem Daumen und dem Zeigefinger die Köpfe des Hebels und des Fixierhebels. zusammendrücken, wodurch sich das Instrumentöffnet(Abb.6). 
Ein äußerst wichtiges Postulat ist die gute, genaue Anästhesie. Der Kehlkopf, aber auch der Zungengrund muß tüchtig kokainisiert werden. Die einfache Pinselung ist nicht immer ausreichend, ein Kokainspray ist kaum zu entbehren.

Vorteile des Verfahrens sind:

I. Die Glottis ist ad maximum erweitert, wodurch der Einblick ins Kehlkopfinnere sehr erleichtert ist. Hier muß ich darauf hinweisen, daß die Distraktion auch bei der genauen Kehllkopfuntersuchung sehr wichtige Dienste leisten kann. Hauptsächlich kann die untere Fläche der Stimmbänder genau betrachtet werden, wenn wir den Gerberschen subglottischen Spiegel benützen. Da die Glottis sich nicht schließen kann, ist mit dem Gerberschen Verfahren diese Region besonders gut betrachtbar.

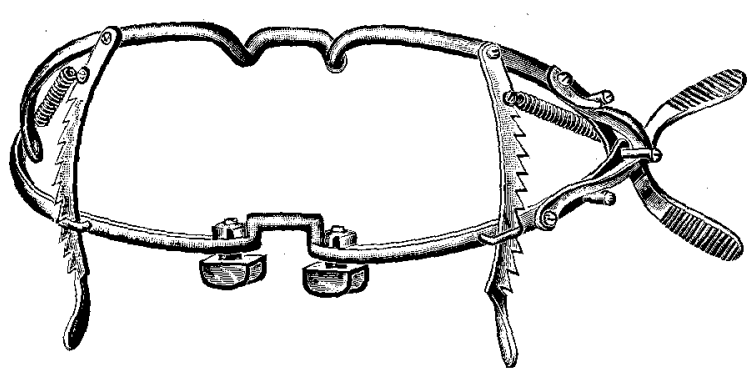

Abb. 5 .

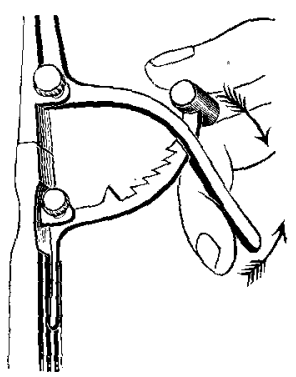

Abb. 6.

2. Die Stimmbänder sind fixiert und angespannt. Das ist überhaupt, aber besonders bei feineren operativen Eingriffen äußerst wichtig. Wir müssen nur daran denken, wie unangenehm es ist, wenn die Stimmbänder sich sehr bewegen, sich einander pressen, wenn wir bei einem Sänger ein Sängerknötchen entfernen müssen. Überhaupt kann ein operatives Vorgehen dadurch ganz verhindert werden. Außer dex Fixation trägt die Anspannung der Stimmbänder noch dazu bei, daß die Operationen an den Stimmbändern leichter werden.

3. Die Epiglottis ist nach vorne gedrückt, wodurch die ganze vordere Partie des Kehlkopfeinganges freigemacht wird. Da der Druck hauptsächlich die Epiglottiswurzel betrifft, so wird die Comissura ant. tadellos sichtbar.

4. Die Zunge zu halten, ist unnötig geworden, da sie durch das Instrument hinuntergedrückt wird. Das ist hauptsächlich bei den sehr fleischigen Zungen ein großer Vorteil, da diese oft große Schwierigkeiten verursachen; anderseits ist aber die oft ungeschickte Hand des Patienten nicht im Wege. 
5. Durch die Mundsperre ist auch der Mund fixiert und offengehalten. Dadurch ist der ganze Einblick überraschend frei geworden. Während bei den größeren Veränderungen die Schwebelaryngoskopie in Anspruch genommen wird, erleichtert bei den kleineren Veränderungen unsere Aufgabe die Larynxdistraktion. Der lebende Larynx wird dadurch, wie gesagt, in ein Phantom umgewandelt.

Das Instrument ist in zwei Größen vorhanden. Der Griff ist gemeinsam.

Fabrikant des Distraktors ist die Firma: Reiner u. Lieberknecht, Wien. 